\title{
Studies on the synthesis of Strychnos indole alkaloids from 2-(3-indolyl)piperidine derivatives. A new synthetic entry to the indolo[3,2-a]quinolizidine system
}

\author{
Mercedes Amat,* Nuria Llor, $\mathbf{M}^{\mathrm{a}}$ Dolors Coll, Nuria Casamitjana, and Joan Bosch* \\ Laboratory of Organic Chemistry, Faculty of Pharmacy, University of Barcelona, Av. Joan \\ XXIII s/n, Barcelona 08028, Spain \\ E-mail: amat@farmacia.far.ub.es
}

This work is dedicated to Prof. Enrique Meléndez on the occasion of his $70^{\text {th }}$ birthday

(received 05 May 03; accepted 18 Jun 03; published on the web 10 Jul 03)

\begin{abstract}
The attempted elaboration of the pentacyclic skeleton of Strychnos indole alkaloids by a Pummerer-initiated sequential closure of $\mathrm{C}$ and $\mathrm{E}$ rings from a 2-(3-indolyl)piperidine-4-acetate derivative bearing a 2-(phenylsulfinyl)ethyl chain on the piperidine nitrogen is described. An indolo[3,2-a]quinolizidine, resulting from the direct attack of the intermediate thionium ion on the 2 position of the heterocycle, is obtained instead.
\end{abstract}

Keywords: Indoloquinolizidine, Pummerer reaction, palladium(0)-cross coupling, electrophilic substitution on indoles, Strychnos alkaloids

\section{Introduction}

In previous papers we have described the preparation of 2-(2-indolyl)- and 2-(3indolyl)piperidine derivatives from the corresponding 2-(indolyl)pyridines, which were efficiently obtained by palladium(0)-catalyzed heteroarylation of 2- and 3-indolylzinc chlorides with 2-halopyridines. These indolylpiperidines have served as synthetic precursors of indolo[2,3a]quinolizidines and alkaloids of the uleine group. ${ }^{1}$ In order to explore new synthetic applications of 2-(3-indolyl)piperidine derivatives, we devised a synthetic approach to the pentacyclic skeleton of Strychnos indole alkaloids ${ }^{2}$ involving the sequential closure of $\mathrm{C}$ and $\mathrm{E}$ rings in a single synthetic step from a 2-(3-indolyl)piperidine-4-acetate derivative bearing a functionalized two-carbon chain on the piperidine nitrogen atom (Figure 1). 

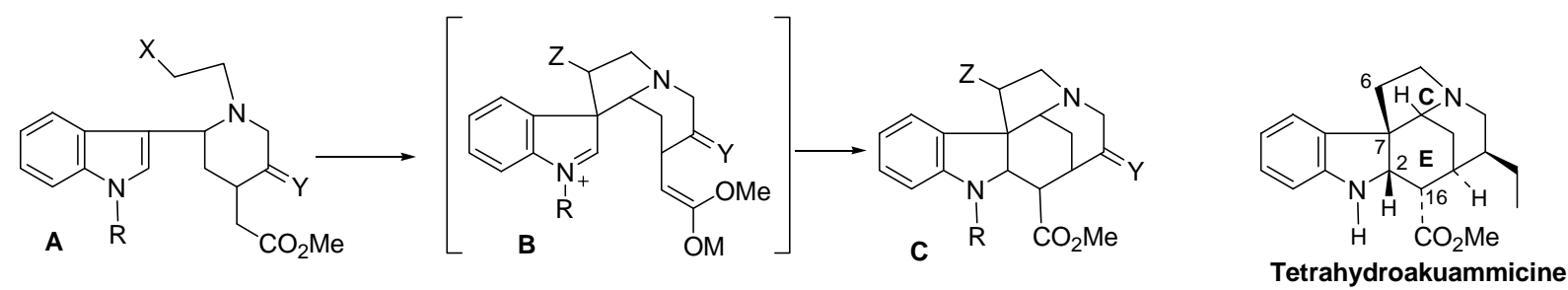

Figure 1

For this purpose, taking into account our previous experience ${ }^{1,3}$ in the incorporation of the tryptamine two-carbon atoms, present in many indole alkaloids, by intramolecular alkylation of the indole 3-position from $N$-[2-(phenylsulfinyl)ethyl] indolylpiperidine derivatives, we selected the trimethylsilyl trifluoromethanesulfonate (TMSOTf)-promoted ${ }^{4}$ Pummerer $^{5}$ reaction of sulfoxide $\mathrm{A}\left(\mathrm{X}=\mathrm{S}(\mathrm{O}) \mathrm{C}_{6} \mathrm{H}_{5} ; \mathrm{R}=\mathrm{CO}_{2} \mathrm{Me}, \mathrm{Y}=\mathrm{CHMe}\right)$. Thus, the intramolecular attack on the indole 3-position by the thionium ion generated in the Pummerer reaction would afford an intermediate $N$-acyl spiroindoleninium salt $\mathbf{B}\left(\mathrm{Z}=\mathrm{SC}_{6} \mathrm{H}_{5}, \mathrm{R}=\mathrm{CO}_{2} \mathrm{Me}\right)$, which could be trapped by the $O$-silyl ketene acetal $\left(\mathrm{M}=\mathrm{SiMe}_{3}\right)$ formed in situ under the reaction conditions [TMSOTf and diisopropylethylamine (DIPEA)]. ${ }^{6}$ Two possible mechanistic pathways have been proposed for the electrophilic substitution in 3-substituted indoles: ${ }^{7}$ either the direct attack at the indole 2position or the attack at the 3-position of the indole ring followed by rearrangement of the resulting indolenine intermediate. However, the studies conducted by Jackson, ${ }^{8}$ as well as the numerous mechanistic studies about the Pictet-Spengler ${ }^{9}$ and Bischler-Napieralski ${ }^{10}$ reactions, have provided a substantial amount of evidence in support of the spiroindolenine intermediate. ${ }^{11}$ Furthermore, several reports have described the intramolecular trapping of the spiroindoleninium intermediate by a nucleophilic residue to furnish polycyclic spiroindolines. ${ }^{12,13}$ In this context, it is worth mentioning that sequential treatment of $\mathrm{N}$-(2-hydroxyethyl)-2-(1-benzenesulfonyl-3indolyl)piperidine-4-acetate $\mathbf{A}\left(\mathrm{X}=\mathrm{OH} ; \mathrm{R}=\mathrm{SO}_{2} \mathrm{C}_{6} \mathrm{H}_{5} ; \mathrm{Y}=\mathrm{H}, \mathrm{Et}\right)$ with $t$-BuOK and $\mathrm{BF}_{3} \cdot \mathrm{Et}_{2} \mathrm{O}$ affords a mixture of the alkaloid tetrahydroakuammicine and the indolo[2,3-a]quinolizidine resulting from the rearrangement of the intermediate spiroindolenine, ${ }^{14}$ which would corroborate the feasibility of our synthetic route to Strychnos alkaloids.

\section{Results and Discussion}

For the preparation of the required 2-(3-indolyl)piperidine-4-acetate derivative 6 we envisaged a synthetic route involving as the key steps a cross-coupling reaction for the generation of the 2-(3indolyl)pyridine moiety and a Johnson-Claisen rearrangement ${ }^{15}$ for the incorporation of the acetate chain at the piperidine 4-position. Thus, 3-(2-pyridyl)indole 3 was obtained in $72 \%$ yield by palladium(0)-catalyzed cross-coupling reaction of 3-indolylzinc chloride $\mathbf{1}$ with 2chloropyridine 2 , followed by desilylation of the indole nitrogen with tetrabutylammonium fluoride (TBAF) (Scheme 1). Methanolysis of acetate 3 afforded alcohol 4 in good yield, which 
on treatment with benzyl bromide followed by $\mathrm{NaBH}_{4}$ reduction of the resulting pyridinium salt gave 2-(3-indolyl)tetrahydropyridine 5 as a nearly equimolecular mixture of epimers. ${ }^{16}$ JohnsonClaisen rearrangement of the allylic alcohol 5 with methyl orthoacetate gave access to 2-(3indolyl)piperidine-4-acetate $\mathbf{6}$ in $72 \%$ yield as a mixture of 2,4 cis and trans isomers, which could not be separated. Unfortunately, all attempts to remove the benzyl protecting group from compound 6 or from its $N$-(methoxycarbonyl)indole derivative 7 by catalytic hydrogenation resulted in failure, the reduction of the ethylidene side chain being the only process observed. On the other hand, treatment of compound 7 with benzyl chloroformate in refluxing dichloromethane afforded compound $\mathbf{8}$ as the only identifiable product, whereas no reaction was observed at room temperature. Compound $\mathbf{8}$ is probably formed by cleavage of the $\mathrm{N}-\mathrm{C}_{2}$ bond from the intermediate $\mathrm{N}$-acyl ammonium salt, with the assistance of the indole, to generate a 3alkylideneindoleninium salt, which undergoes deprotonation.

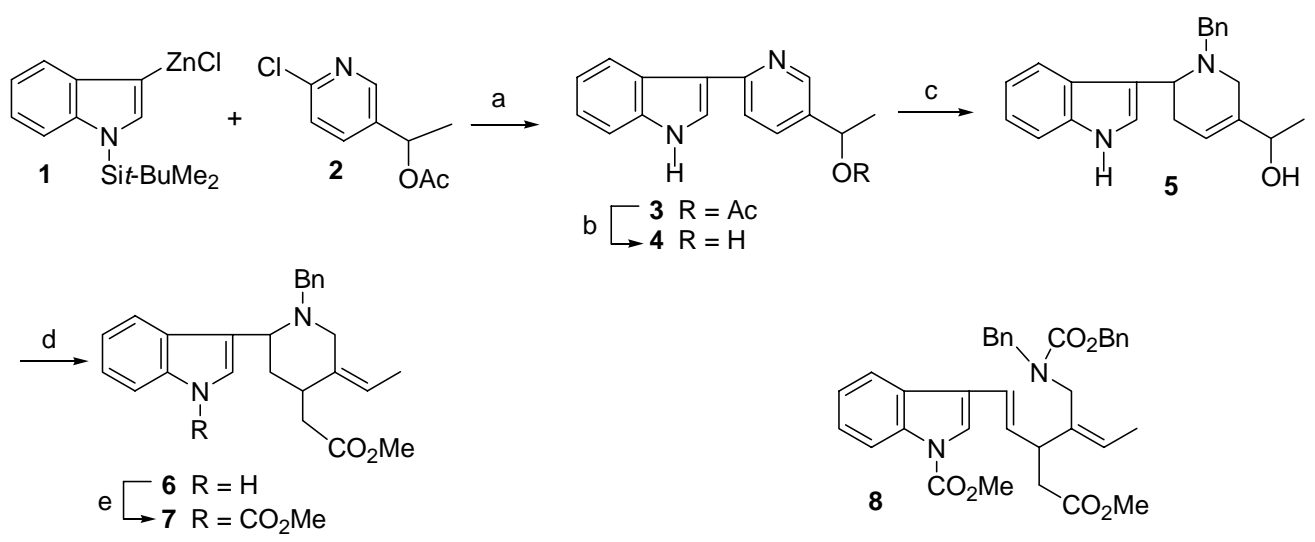

Scheme 1. Reagents and conditions: (a) $\mathrm{Pd}\left[\mathrm{P}\left(\mathrm{C}_{6} \mathrm{H}_{5}\right)_{3}\right]_{4}$, DIBAH, THF, reflux, $4 \mathrm{~h}$, then TBAF, THF, $25{ }^{\circ} \mathrm{C}, 15 \mathrm{~min}, 72 \%$; (b) $\mathrm{K}_{2} \mathrm{CO}_{3}, \mathrm{MeOH}, 25{ }^{\circ} \mathrm{C}, 1 \mathrm{~h}, 95 \%$; (c) BrBn, $\mathrm{C}_{6} \mathrm{H}_{6}$-acetone, reflux, $24 \mathrm{~h}$, then $\mathrm{NaBH}_{4}, \mathrm{MeOH}, 25^{\circ} \mathrm{C}, 12 \mathrm{~h}, 67 \%$; (d) $\mathrm{CH}_{3} \mathrm{C}(\mathrm{OMe})_{3}$, dimethoxyethane, pivalic acid, reflux, $48 \mathrm{~h}, 64 \%$; (e) $\mathrm{NCCO}_{2} \mathrm{Me}$, DMAP, $\mathrm{CH}_{3} \mathrm{CN}$, reflux, $5 \mathrm{~h}, 91 \%$.

The difficulties encountered in the removal of the $N$-benzyl group from $\mathbf{6}$ or $\mathbf{7}$ made us go back in the synthetic sequence and incorporate the functionalized two-carbon chain on the piperidine nitrogen atom at an early stage of the synthesis. Thus, compound 4 was treated with 2iodoethyl phenyl sulfide and the resulting pyridinium salt was subsequently reduced with sodium borohydride to give the desired tetrahydropyridine $\mathbf{9}$ as a mixture of stereoisomers (Scheme 2). Johnson-Claisen rearrangement of the allylic alcohol 9 satisfactorily gave the 2-(3indolyl)piperidine-4-acetate $\mathbf{1 0}$ as a nearly equimolecular mixture of cis and trans isomers. On the grounds of our previous experience in the Pummerer reaction on indole derivatives, ${ }^{1 \mathrm{c}, 3}$ we decided to prepare compound 11, which bears an electron-withdrawing protecting group on the indole nitrogen. Moreover, acylation of the indole nitrogen could facilitate the closure of the $\mathrm{E}$ ring, since a highly reactive $\mathrm{N}$-acyliminium intermediate $\mathbf{B}$ (Figure $1, \mathrm{R}=\mathrm{CO}_{2} \mathrm{Me}$ ) would be generated after the attack of the thionium ion on the indole 3-position. Compound $\mathbf{1 1}$ was 
obtained by treatment of $\mathbf{1 0}$ with methyl cyanoformate and 4-(dimethylamino)pyridine (DMAP) in refluxing acetonitrile. Chemoselective oxidation of sulfide 11 with 3-chloroperbenzoic acid ( $m$-CPBA) was carried out from the corresponding trifluoroacetate salt at low temperature in order to avoid oxidation of the nitrogen and double bond functions. In this way, sulfoxide 12 was isolated in 72\% yield. Finally, treatment of $\mathbf{1 2}$ with excess (4 equiv) TMSOTf and DIPEA in dichloromethane at room temperature gave tetracyclic indolo[3,2-a]quinolizidine $\mathbf{1 3}$ as a nearly equimolecular mixture of isomers 13a and $\mathbf{1 3 b}$ in $42 \%$ yield. Neither the desired pentacyclic compound with the Strychnos skeleton nor the corresponding indolo[2,3-a]quinolizidine resulting from the rearrangement of the presumed spiroindoleninium intermediate could be detected, which clearly indicates that the electrophilic attack of the thionium ion generated from sulfoxide 12 under Pummerer conditions does not take place at the indole 3-position but directly at the 2-position of the heterocycle. This result can be accounted for by considering previous studies by Casnati ${ }^{17}$ who has shown that the alkylation of 3 -substituted indoles can occur by direct attack at the 2-position of the indole when very reactive electrophiles are involved.

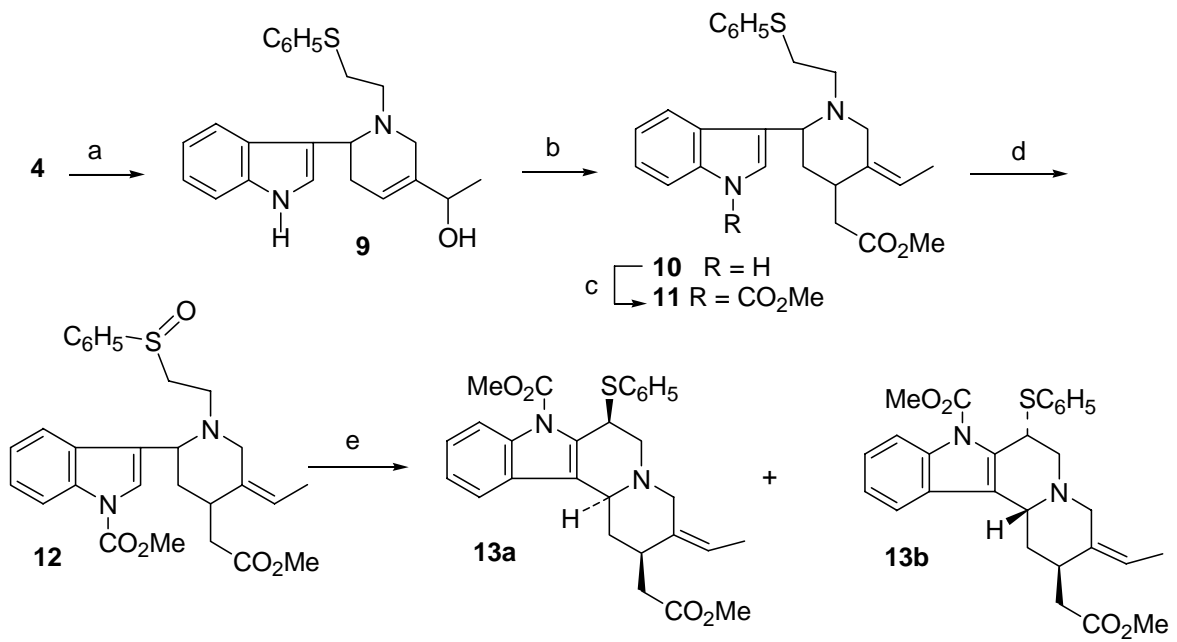

Scheme 2. Reagents and conditions: (a) $\mathrm{ICH}_{2} \mathrm{CH}_{2} \mathrm{SC}_{6} \mathrm{H}_{5}, \mathrm{MeOH}$, reflux, overnight, then $\mathrm{NaBH}_{4}$, $\mathrm{MeOH}, 25^{\circ} \mathrm{C}, 4 \mathrm{~h}, 45 \%$; (b) $\mathrm{CH}_{3} \mathrm{C}(\mathrm{OMe})_{3}$, dimethoxyethane, pivalic acid, reflux, $48 \mathrm{~h}, 69 \%$; (c) $\mathrm{NCCO}_{2} \mathrm{Me}$, DMAP, $\mathrm{CH}_{3} \mathrm{CN}$, reflux, $5 \mathrm{~h}, 74 \%$; (d) TFA, $\mathrm{CH}_{2} \mathrm{Cl}_{2}, 0^{\circ} \mathrm{C}, 30$ min, then $\mathrm{MCPBA}, \mathrm{CH}_{2} \mathrm{Cl}_{2}$, $-60^{\circ} \mathrm{C}, 2 \mathrm{~h}, 72 \%$; (e) TMSOTf, DIPEA, $\mathrm{CH}_{2} \mathrm{Cl}_{2}, 0^{\circ} \mathrm{C}, 1.5 \mathrm{~h}, 42 \%$.

It is worth mentioning that the conversion of indole alkaloids of the indolo[2,3a]quinolizidine type into the corresponding indolo[3,2-a]quinolizidine derivatives ("inverted" alkaloids) has been described previously by sodium borohydride reduction of the corresponding pseudoindoxyl analogs followed by treatment of the product under acidic conditions. ${ }^{18}$ 


\section{Conclusions}

In summary, the electrophilic attack of the thionium ion generated by Pummerer rearrangement of the sulfoxide present in 2-(3-indolyl)- $N$-2-(phenylsulfinyl)ethylpiperidine derivatives such as 12, takes place directly on the unsubstituted indole 2-position affording an indolo[3,2a]quinolizidine system.

\section{Experimental Section}

General Procedures. Melting points were determined on a Gallenkamp melting point apparatus and are uncorrected. NMR spectra were recorded on either a Varian Gemini 200 operating at 200 $\mathrm{MHz}$ for ${ }^{1} \mathrm{H}$ and $50.3 \mathrm{MHz}$ for ${ }^{13} \mathrm{C}$, or a Varian Gemini 300 operating at $300 \mathrm{MHz}$ for ${ }^{1} \mathrm{H}$ and $75.5 \mathrm{MHz}$ for ${ }^{13} \mathrm{C}$. Chemical shifts are reported in values ppm relative to TMS as internal reference. IR spectra were recorded on a FTIR Perkin-Elmer 1600 spectrometer with samples prepared either as $\mathrm{KBr}$ pellets or thin films on $\mathrm{NaCl}$ salt plates, and only noteworthy absorptions are listed. Thin-layer chromatography was done on $\mathrm{SiO}_{2}$ (silica gel $60 \mathrm{~F}_{254}$, Merck), and the spots were located with aqueous potassium permanganate solution. Column chromatography was carried out on $\mathrm{SiO}_{2}$ (silica gel 60, SDS, 70-200 microns). Flash chromatography was carried out on $\mathrm{SiO}_{2}$ (silica gel 60, SDS, 35-70 microns). All reagents were purchased from Aldrich or Fluka and were used without further purification. Tetrahydrofuran was distilled from sodium/benzophenone. Solvents for chromatography were distilled at atmospheric pressure prior to use and dried using standard procedures. All reactions were performed under argon or nitrogen. Drying of the organic extracts during the work-up of reactions was performed over $\mathrm{Na}_{2} \mathrm{SO}_{4}$. Evaporation of solvents was accomplished with a rotatory evaporator. Microanalyses were performed by Centre d'Investigació i Desenvolupament (CSIC), Barcelona.

5-(1-Acetoxyethyl)-2-(3-indolyl)pyridine (3). A solution of $t$-BuLi (7.6 mL of a $1.7 \mathrm{M}$ solution in pentane, $13.0 \mathrm{mmol}$ ) was slowly added to a solution of 3-bromo-1-(tertbutyldimethylsilyl)indole $(2.0 \mathrm{~g}, 6.45 \mathrm{mmol})$ in anhydrous THF $(5 \mathrm{~mL})$ at $-78{ }^{\circ} \mathrm{C}$, and the resulting mixture was stirred for $10 \mathrm{~min}$ at this temperature. Then, a solution of anhydrous $\mathrm{ZnCl}_{2}$ (886 mg, $6.5 \mathrm{mmol})$ in THF (4 mL) was added, and the stirring was continued for $30 \mathrm{~min}$ at $25^{\circ} \mathrm{C}$. In a separate flask, a solution of $\operatorname{Pd}\left[\mathrm{P}\left(\mathrm{C}_{6} \mathrm{H}_{5}\right)_{3}\right]_{4}(499 \mathrm{mg}, 0.43 \mathrm{mmol})$ in anhydrous THF $(16 \mathrm{~mL})$ was added to a solution of chloropyridine $2(862 \mathrm{mg}, 4.32 \mathrm{mmol})$ in THF $(8 \mathrm{~mL})$, and the mixture was stirred at room temperature for $10 \mathrm{~min}$. The resulting solution was transferred via cannula to the solution of indolylzinc $\mathbf{1}$ in THF prepared as described above, and the mixture was heated at reflux for $4 \mathrm{~h}$, cooled, and poured into saturated aqueous $\mathrm{Na}_{2} \mathrm{CO}_{3}$. The aqueous layer was extracted with $\mathrm{Et}_{2} \mathrm{O}$, and the combined organic extracts were dried, filtered, and concentrated to give a residue, which was subjected to desilylation without further purification. A 1.0 M solution of tetrabutylammonium fluoride $(6.5 \mathrm{~mL}, 6.5 \mathrm{mmol})$ was added to a solution of 
the above reaction mixture in anhydrous THF $(22 \mathrm{~mL})$, and the mixture was stirred for $15 \mathrm{~min}$ at room temperature. The solution was poured into saturated aqueous $\mathrm{Na}_{2} \mathrm{CO}_{3}$, and the aqueous layer was extracted with $\mathrm{CH}_{2} \mathrm{Cl}_{2}$. The combined organic extracts were dried, filtered, and concentrated. The residue was chromatographed (gradient from 2:3 AcOEt-hexane to AcOEt) to give pure indolylpyridine 3 (741 mg, 72\%). IR (KBr) 3300, 1731, $1240 \mathrm{~cm}^{-1}$; ${ }^{1} \mathrm{H} \mathrm{NMR}\left(\mathrm{CDCl}_{3}\right.$, $200 \mathrm{MHz}) \delta 1.61(\mathrm{~d}, J=6.6 \mathrm{~Hz}, 3 \mathrm{H}), 2.1(\mathrm{~s}, 3 \mathrm{H}), 5.94(\mathrm{q}, J=6.6 \mathrm{~Hz}, 1 \mathrm{H}), 7.27(\mathrm{~m}, 2 \mathrm{H}), 7.41$ $(\mathrm{m}, 1 \mathrm{H}), 7.70(\mathrm{~m}, 2 \mathrm{H}), 7.77(\mathrm{~d}, J=2.6 \mathrm{~Hz}, 1 \mathrm{H}), 8.32(\mathrm{~m}, 1 \mathrm{H}), 8.61$ (br s, $1 \mathrm{H}), 8.67(\mathrm{~m}, 1 \mathrm{H})$; ${ }^{13} \mathrm{C} \mathrm{NMR}\left(\mathrm{CDCl}_{3}, 75 \mathrm{MHz}\right) \delta 21.2\left(\mathrm{CH}_{3}\right), 21.7\left(\mathrm{CH}_{3}\right), 70.3(\mathrm{CH}), 111.6(\mathrm{CH}), 116.3(\mathrm{C}), 120.2$ $(\mathrm{CH}), 120.5(\mathrm{CH}), 120.7(\mathrm{CH}), 122.3(\mathrm{CH}), 125.0(\mathrm{CH}), 125.1(\mathrm{C}), 133.1(\mathrm{C}), 134.5(\mathrm{CH}), 136.9$ (C), $147.4(\mathrm{CH}), 154.7(\mathrm{C}), 170.3(\mathrm{C})$; mp 98-99 ${ }^{\circ} \mathrm{C}$ (Et ${ }_{2} \mathrm{O}-$ hexane). Anal. Calcd for $\mathrm{C}_{17} \mathrm{H}_{16} \mathrm{~N}_{2} \mathrm{O}_{2}$ : C, 72.83; H, 5.75; N, 9.99. Found: C, 72.89; H, 5.74; N, 10.00 .

5-(1-Hydroxyethyl)-2-(3-indolyl)pyridine (4). A mixture of acetate 3 (0.83 g, $2.96 \mathrm{mmol})$ and $\mathrm{K}_{2} \mathrm{CO}_{3}(1.23 \mathrm{~g}, 8.9 \mathrm{mmol})$ in absolute $\mathrm{MeOH}(8 \mathrm{~mL})$ was stirred for $1 \mathrm{~h}$ at room temperature. The solvent was eliminated under vacuum, and the residue was dissolved in $\mathrm{CH}_{2} \mathrm{Cl}_{2}$, washed with brine, dried, filtered, and concentrated. The residue was chromatographed (AcOEt) to give pure alcohol 4 (670 mg, 95\%). IR (KBr) 3168, $1601 \mathrm{~cm}^{-1} ;{ }^{1} \mathrm{H}$ NMR $\left(\mathrm{CD}_{3} \mathrm{OD}, 300 \mathrm{MHz}\right) \delta 1.50$ $(\mathrm{d}, J=6.5 \mathrm{~Hz}, 3 \mathrm{H}), 4.89(\mathrm{q}, J=6.5 \mathrm{~Hz}), 7.25(\mathrm{~m}, 2 \mathrm{H}), 7.54(\mathrm{dm}, J=7.2 \mathrm{~Hz}, 1 \mathrm{H}), 7.87(\mathrm{dd}, J=$ 8.3, $0.9 \mathrm{~Hz}, 1 \mathrm{H}), 7.89$ (s, $1 \mathrm{H}), 7.92$ (dd, $J=8.3,2.2 \mathrm{~Hz}, 1 \mathrm{H}), 8.21$ (dm, $J=7.8 \mathrm{~Hz}, 1 \mathrm{H}), 8.62$ $(\mathrm{m}, 1 \mathrm{H}) ;{ }^{13} \mathrm{C}$ NMR $\left(\mathrm{CD}_{3} \mathrm{OD}, 75 \mathrm{MHz}\right) \delta 25.3\left(\mathrm{CH}_{3}\right), 68.5(\mathrm{CH}), 112.7(\mathrm{CH}), 116.9(\mathrm{C}), 121.2$ $(\mathrm{CH}), 121.3(\mathrm{CH}), 122.0(\mathrm{CH}), 123.1(\mathrm{CH}), 126.2(\mathrm{CH}), 126.6(\mathrm{C}), 135.7(\mathrm{CH}), 138.7(\mathrm{C}), 139.3$ (C), $147.6(\mathrm{CH}), 155.7(\mathrm{C}) ; \mathrm{mp} 176^{\circ} \mathrm{C}(\mathrm{MeOH})$. Anal. Calcd for $\mathrm{C}_{15} \mathrm{H}_{14} \mathrm{~N}_{2} \mathrm{O}$ : C, 75.60; H, 5.92; N, 11.75. Found: C, 75.63; H, 5.91; N, 11.83 .

\section{1-Benzyl-5-(1-hydroxymethyl)-2-(3-indolyl)-1,2,3,6-tetrahydropyridine (mixture of isomers)}

(5). Benzyl bromide $(1.1 \mathrm{~mL}, 9.26 \mathrm{mmol}$ ) was added to a solution of indolylpyridine 4 (940 $\mathrm{mg}$, $3.95 \mathrm{mmol})$ in a 2:1 mixture of anhydrous benzene and acetone $(9 \mathrm{~mL})$, and the solution was refluxed for $24 \mathrm{~h}$. The mixture was concentrated under vacuum, and the resulting pyridinium salt was washed with anhydrous $\mathrm{Et}_{2} \mathrm{O}$, dried, and dissolved in $\mathrm{MeOH}(40 \mathrm{~mL})$. The solution was cooled to $0{ }^{\circ} \mathrm{C}, \mathrm{NaBH}_{4}(625 \mathrm{mg}, 16.5 \mathrm{mmol})$ was added, and the stirring was continued at room temperature for $12 \mathrm{~h}$. The solvent was eliminated under vacuum, and the residue was dissolved in a 1:1 mixture of $\mathrm{Et}_{2} \mathrm{O}-\mathrm{H}_{2} \mathrm{O}(100 \mathrm{~mL}) . \mathrm{K}_{2} \mathrm{CO}_{3}(600 \mathrm{mg})$ was added, and the resulting mixture was stirred for $1 \mathrm{~h}$. The aqueous layer was washed with $\mathrm{Et}_{2} \mathrm{O}$, and the combined organic extracts were dried, filtered, and concentrated. The residue was chromatographed $\left(\mathrm{Et}_{2} \mathrm{O}\right)$ to give the tetrahydropyridine 5 ( $878 \mathrm{mg}, 67 \%$ ) as a 55:45 mixture of isomers (calculated by ${ }^{1} \mathrm{H} \mathrm{NMR}$ ), which could not be separated. IR (film) 3000, 1455, $743 \mathrm{~cm}^{-1} ;{ }^{1} \mathrm{H} \mathrm{NMR}\left(\mathrm{CDCl}_{3}, 300 \mathrm{MHz}\right.$, mixture of epimers) $\delta 1.26(\mathrm{~d}, J=6.4 \mathrm{~Hz}, 3 \mathrm{H}), 1.27(\mathrm{~d}, J=6.4 \mathrm{~Hz}, 3 \mathrm{H}), 1.60$ (br s, $1 \mathrm{H}$ each isomer); 2.40-2.58 (m, $1 \mathrm{H}$ each isomer); 2.62-2.58 (m, $1 \mathrm{H}$ each isomer), $2.94(\mathrm{dm}, J=16.6 \mathrm{~Hz}$, $1 \mathrm{H}$ each isomer), $3.15(\mathrm{~d}, J=16.6 \mathrm{~Hz}, 1 \mathrm{H}), 3.17(\mathrm{~d}, J=13.0 \mathrm{~Hz}, 1 \mathrm{H}), 3.22(\mathrm{~d}, J=13.0 \mathrm{~Hz}, 1$ H), $3.226(\mathrm{~d}, J=16.6 \mathrm{~Hz}, 1 \mathrm{H}), 3.77$ (d, $J=13.0 \mathrm{~Hz}, 1 \mathrm{H}), 3.79$ (d, $J=13.0 \mathrm{~Hz}, 1 \mathrm{H}), 4.02$ (dd, $J$ $=8.5,5.0 \mathrm{~Hz}, 1 \mathrm{H}), 4.07(\mathrm{dd}, J=8.0,5.5 \mathrm{~Hz}, 1 \mathrm{H}), 4.21(\mathrm{q}, J=6.4 \mathrm{~Hz}, 1 \mathrm{H}$ each isomer), 5.84 (br s, $1 \mathrm{H}$ each isomer), 7.10-7.37 (m), $7.36(\mathrm{~d}, J=7.8 \mathrm{~Hz}, 1 \mathrm{H}$ each isomer), $7.87(\mathrm{~m}), 8.15$ (br s); ${ }^{13} \mathrm{C} \mathrm{NMR}\left(\mathrm{CDCl}_{3}, 75 \mathrm{MHz}\right.$, mixture of epimers $) \delta 21.8\left(\mathrm{CH}_{3}\right), 21.9\left(\mathrm{CH}_{3}\right), 32.6\left(\mathrm{CH}_{2}\right), 32.8$ 
$\left(\mathrm{CH}_{2}\right), 50.0\left(\mathrm{CH}_{2}\right), 50.3\left(\mathrm{CH}_{2}\right), 55.2(\mathrm{CH}), 55.4(\mathrm{CH}), 58.8\left(\mathrm{CH}_{2}\right), 58.9\left(\mathrm{CH}_{2}\right), 69.7(\mathrm{CH}), 70.0$ $(\mathrm{CH}), 111.1(2 \mathrm{CH}), 116.5(2 \mathrm{C}), 118.8(2 \mathrm{CH}), 119.3(2 \mathrm{CH}), 119.7(2 \mathrm{CH}), 121.9(2 \mathrm{CH}), 122.3$ $(\mathrm{CH}), 122.4(\mathrm{CH}), 126.7(2 \mathrm{CH}), 127.1(2 \mathrm{C}), 128.0(4 \mathrm{CH}), 129.1(2 \mathrm{CH}), 129.2(2 \mathrm{CH}), 136.1$ (2 C), 138.8 (C), 139.0 (C), 139.6 (C), 139.8 (C). Exact mass Calcd for $\mathrm{C}_{22} \mathrm{H}_{24} \mathrm{~N}_{2} \mathrm{O}: 332.1888$. Found: 332.1880 .

Methyl 1-benzyl-5(Z)-ethylidene-2-(3-indolyl)-4-piperidineacetate (mixture of isomers) (6). Methyl orthoacetate $(1.5 \mathrm{~mL}, 11.9 \mathrm{mmol})$ and pivalic acid $(13 \mathrm{mg}, 0.12 \mathrm{mmol})$ were added to a solution of tetrahydropyridine 5 (400 $\mathrm{mg}, 1.2 \mathrm{mmol}$, mixture of epimers) in anhydrous dimethoxyethane $(7 \mathrm{~mL})$, and the mixture was heated at reflux for $48 \mathrm{~h}$. The solvent was eliminated under reduced pressure, the residue was dissolved in $\mathrm{CH}_{2} \mathrm{Cl}_{2}$, and the solution was washed with saturated aqueous $\mathrm{Na}_{2} \mathrm{CO}_{3}$, dried, filtered, and concentrated. The resulting residue was chromatographed (1:3 AcOEt-hexane) to give compound 6 (300 mg, 64\%) as a nearly equimolecular mixture of isomers, which could not be efficiently separated. IR (film) 1728, 742 $\mathrm{cm}^{-1}$; cis isomer: ${ }^{1} \mathrm{H} \mathrm{NMR}\left(\mathrm{CDCl}_{3}, 300 \mathrm{MHz}\right.$, most significant signals from an enriched fraction) $\delta 1.47(\mathrm{~d}, J=6.6 \mathrm{~Hz}, 3 \mathrm{H}), 2.25(\mathrm{dd}, J=14.7,7.4 \mathrm{~Hz}, 1 \mathrm{H}), 2.34(\mathrm{~d}, J=12.5 \mathrm{~Hz}, 1 \mathrm{H}), 2.98(\mathrm{~d}, J$ $=13.4 \mathrm{~Hz}, 1 \mathrm{H}), 3.65(\mathrm{~s}, 3 \mathrm{H}), 3.80(\mathrm{dd}, J=11.2,2.2 \mathrm{~Hz}, 1 \mathrm{H}), 3.84(\mathrm{~d}, J=12.5 \mathrm{~Hz}, 1 \mathrm{H}), 3.96$ $(\mathrm{d}, J=13,4 \mathrm{~Hz}, 1 \mathrm{H}), 5.13(\mathrm{q}, J=6.6 \mathrm{~Hz}, 1 \mathrm{H}), 7.10-7.40(\mathrm{~m}, 9 \mathrm{H}), 7.95(\mathrm{~m}, 1 \mathrm{H}), 8.10$ (br s, 1 $\mathrm{H}) ;{ }^{13} \mathrm{C} \mathrm{NMR}\left(\mathrm{CDCl}_{3}, 75 \mathrm{MHz}\right) \delta 12.6\left(\mathrm{CH}_{3}\right), 36.8\left(\mathrm{CH}_{2}\right), 39.0(\mathrm{CH}), 41.8\left(\mathrm{CH}_{2}\right), 51.6\left(\mathrm{CH}_{3}\right)$, $52.9\left(\mathrm{CH}_{2}\right), 58.8\left(\mathrm{CH}_{2}\right), 61.2(\mathrm{CH}), 111.1(\mathrm{CH}), 114.6(\mathrm{CH}), 118.4(\mathrm{C}), 119.2(\mathrm{CH}), 120.2(\mathrm{CH})$, $122.0(\mathrm{CH}), 126.4(\mathrm{CH}), 128.0(2 \mathrm{CH}), 128.5(2 \mathrm{CH}), 128.8(\mathrm{C}), 135.9(\mathrm{C}), 137.5(\mathrm{C}), 138.8(\mathrm{C})$, 173.6 (C); trans isomer: ${ }^{1} \mathrm{H}$ NMR $\left(\mathrm{CDCl}_{3}, 300 \mathrm{MHz}\right.$, most significant signals from an enriched fraction) $\delta 1.43(\mathrm{~d}, J=6.6 \mathrm{~Hz}, 3 \mathrm{H}), 3.08(\mathrm{~d}, J=13.4 \mathrm{~Hz}, 1 \mathrm{H}), 3.60(\mathrm{~d}, J=13.0 \mathrm{~Hz}, 1 \mathrm{H}), 3.65$ $(\mathrm{s}, 3 \mathrm{H}), 3.95(\mathrm{~d}, J=13.4 \mathrm{~Hz}, 1 \mathrm{H}), 3.97(\mathrm{~m}, 1 \mathrm{H}), 5.38(\mathrm{q}, J=6.6 \mathrm{~Hz}, 1 \mathrm{H}), 7.10-7.40(\mathrm{~m}, 9 \mathrm{H})$, $7.95(\mathrm{~m}, 1 \mathrm{H}), 8.10($ br s, $1 \mathrm{H}) ;{ }^{13} \mathrm{C} \mathrm{NMR}\left(\mathrm{CDCl}_{3}, 75 \mathrm{MHz}\right) \delta 12.7\left(\mathrm{CH}_{3}\right), 37.9\left(\mathrm{CH}_{2}\right), 38.5$ $\left(\mathrm{CH}_{2}\right), 39.6(\mathrm{CH}), 48.0\left(\mathrm{CH}_{2}\right), 51.5\left(\mathrm{CH}_{3}\right), 55.8(\mathrm{CH}), 58.5\left(\mathrm{CH}_{2}\right), 111.1(\mathrm{CH}), 118.4(\mathrm{C}), 119.2$ $(\mathrm{CH}), 119.6(\mathrm{CH}), 120.1(\mathrm{CH}), 122.0(\mathrm{CH}), 126.5(\mathrm{CH}), 128.0(2 \mathrm{CH}), 128.6(2 \mathrm{CH}), 128.8(\mathrm{C})$, 136.4 (C), 137.5 (C), 139.7 (C), 173.1 (C). Exact mass Calcd for $\mathrm{C}_{25} \mathrm{H}_{28} \mathrm{~N}_{2} \mathrm{O}_{2}$ : 388.2150. Found: 388.2144 .

Methyl 1-benzyl-5(Z)-ethylidene-2-(1-methoxycarbonyl-3-indolyl)-4-piperidineacetate (mix ture of isomers) (7). Methyl cyanoformate $(178 \mu \mathrm{L}, 2.24 \mathrm{mmol})$ and 4-(dimethylamino)pyridine (35 mg, $0.29 \mathrm{mmol}$ ) were added to a solution of compound 6 (100 mg, $0.26 \mathrm{mmol}$, mixture of epimers) in acetonitrile $(2 \mathrm{~mL})$, and the mixture was heated at reflux for $5 \mathrm{~h}$, cooled, and concentrated under reduced pressure. The residue was dissolved in $\mathrm{CH}_{2} \mathrm{Cl}_{2}$, and the solution was washed with brine, dried, filtered, and concentrated. The residue was chromatographed (7:3 hexane-AcOEt) to give compound 7 (105 mg, 91\%). ${ }^{1} \mathrm{H} \mathrm{NMR}\left(\mathrm{CDCl}_{3}, 300 \mathrm{MHz}\right.$, most significant signals from a mixture of isomers) $\delta 1.42(\mathrm{~d}, J=6.6 \mathrm{~Hz}, 3 \mathrm{H}), 1.45(\mathrm{~d}, J=6.6 \mathrm{~Hz}, 3$ H), 1.95 (ddd, $J=12.6,3.8,3.5 \mathrm{~Hz}, 1 \mathrm{H}), 2.24$ (dd, $J=14.8,6.6 \mathrm{~Hz}, 1 \mathrm{H}), 2.32$ (d, $J=11.5 \mathrm{~Hz}, 1$ H), $2.58(\mathrm{dd}, J=14.8,7.6 \mathrm{~Hz}, 1 \mathrm{H}), 2.95(\mathrm{~m}, 1 \mathrm{H}), 3.00(\mathrm{~d}, J=13.4 \mathrm{~Hz}, 1 \mathrm{H}), 3.09$ (d, $J=13.4$ $\mathrm{Hz}, 1 \mathrm{H}), 3.58$ (d, J=13.2 Hz, $1 \mathrm{H}), 3.64(\mathrm{~s}, 3 \mathrm{H}), 3.65(\mathrm{~s}, 3 \mathrm{H}), 3.75$ (dd, $J=11.5,2.8 \mathrm{~Hz}, 1 \mathrm{H})$, $3.82(\mathrm{~d}, J=12.4 \mathrm{~Hz}, 1 \mathrm{H}), 3.88(\mathrm{~d}, J=13.4 \mathrm{~Hz}, 1 \mathrm{H}$ each isomer), $4.01(\mathrm{~s}, 3 \mathrm{H}), 4.02(\mathrm{~s}, 3 \mathrm{H})$, $5.14(\mathrm{q}, J=6.6 \mathrm{~Hz}, \mathrm{CH}), 5.38(\mathrm{q}, J=6.6 \mathrm{~Hz}, 1 \mathrm{H}) ; 7.10-7.39(\mathrm{~m}), 7.60$ (d, $J=6.5 \mathrm{~Hz}, 1 \mathrm{H}$ each 
isomer), $7.95\left(\mathrm{~d}, J=7.0 \mathrm{~Hz}, 1 \mathrm{H}\right.$ each isomer), 8.18 (d, $J=7.5 \mathrm{~Hz}, 1 \mathrm{H}$ each isomer); ${ }^{13} \mathrm{C}$ NMR $\left(\mathrm{CDCl}_{3}, 75 \mathrm{MHz}\right.$, signals of cis isomer from an enriched fraction): $\delta 12.5\left(\mathrm{CH}_{3}\right), 36.7\left(\mathrm{CH}_{2}\right)$, $38.7(\mathrm{CH}), 40.6\left(\mathrm{CH}_{2}\right), 51.4\left(\mathrm{CH}_{3}\right), 52.5\left(\mathrm{CH}_{2}\right), 53.6\left(\mathrm{CH}_{3}\right), 58.6\left(\mathrm{CH}_{2}\right), 60.9(\mathrm{CH}), 115.0(\mathrm{CH})$, $119.8(\mathrm{CH}), 120.8(\mathrm{CH}) 122.6(\mathrm{CH}), 122.9(\mathrm{CH}), 123.7(\mathrm{C}), 124.6(\mathrm{CH}), 126.5(\mathrm{CH}), 127.9(2$ $\mathrm{CH}), 128.4(2 \mathrm{CH}), 128.5(\mathrm{C}), 135.8(\mathrm{C}), 137.0(\mathrm{C}), 139.5(\mathrm{C}), 151.3(\mathrm{C}), 172.8(\mathrm{C}) ;{ }^{13} \mathrm{C}$ NMR $\left(\mathrm{CDCl}_{3}, 75 \mathrm{MHz}\right.$, most significant signals of trans isomer): $\delta 12.6\left(\mathrm{CH}_{3}\right), 37.2\left(\mathrm{CH}_{2}\right), 37.7$ $\left(\mathrm{CH}_{2}\right), 39.2(\mathrm{CH}), 47.6\left(\mathrm{CH}_{2}\right), 51.5\left(\mathrm{CH}_{3}\right), 53.6\left(\mathrm{CH}_{3}\right), 55.8(\mathrm{CH}), 58.3\left(\mathrm{CH}_{2}\right)$.

\section{5-(1-Hydroxyethyl)-2-(3-indolyl)-1-[2-(phenylsulfanyl)ethyl]-1,2,3,6-tetrahydropyridine}

(mixture of isomers) (9). 2-(Phenylthio)ethyl iodide (554 $\mathrm{mg}, 2.1 \mathrm{mmol}$ ) was added to a solution of indolylpyridine $4(200 \mathrm{mg}, 0.84 \mathrm{mmol})$ in absolute $\mathrm{MeOH}(1.3 \mathrm{~mL})$, and the mixture was heated at reflux overnight. The mixture was concentrated, dried under vacuum, and washed with anhydrous $\mathrm{Et}_{2} \mathrm{O}$. The resulting pyridinium salt was redissolved in absolute $\mathrm{MeOH}(8 \mathrm{~mL})$, cooled to $0{ }^{\circ} \mathrm{C}$, and treated with $\mathrm{NaBH}_{4}(130 \mathrm{mg}, 3.44 \mathrm{mmol})$. The mixture was stirred for $4 \mathrm{~h}$ at room temperature and concentrated. The resulting residue was dissolved in a 1:1 mixture of $\mathrm{Et}_{2} \mathrm{O}-\mathrm{H}_{2} \mathrm{O}(21 \mathrm{~mL}), \mathrm{K}_{2} \mathrm{CO}_{3}(127 \mathrm{mg})$ was added, and the mixture was stirred for $1 \mathrm{~h}$. The aqueous layer was washed with $\mathrm{Et}_{2} \mathrm{O}$, and the combined ethereal layers were dried, filtered, and concentrated. The residue was chromatographed $\left(\mathrm{Et}_{2} \mathrm{O}\right)$ to give the tetrahydropyridine $9(142 \mathrm{mg}$, $45 \%)$ as a 5:4 mixture of isomers. IR (film) $3409,1455,1438,1069 \mathrm{~cm}^{-1} ;{ }^{1} \mathrm{H} \mathrm{NMR}\left(\mathrm{CDCl}_{3}, 300\right.$ $\mathrm{MHz}$, most significant signals of a mixture of isomers) $\delta 1.32(\mathrm{~d}, J=6.4 \mathrm{~Hz}, 3 \mathrm{H}), 1.34(\mathrm{~d}, J=$ $6.4 \mathrm{~Hz}, 3 \mathrm{H}), 3.15(\mathrm{~m}, 1 \mathrm{H}), 3.20(\mathrm{~m}, 1 \mathrm{H}), 3.37(\mathrm{~d}, J=16.0 \mathrm{~Hz}, 1 \mathrm{H}), 3.52(\mathrm{~d}, J=16.0 \mathrm{~Hz}, 1 \mathrm{H})$, $3.94(\mathrm{dd}, J=8.7,4.7 \mathrm{~Hz}, 1 \mathrm{H}), 4.02(\mathrm{dd}, J=7.7,5.0 \mathrm{~Hz}, 1 \mathrm{H}), 4.30$ (m, $1 \mathrm{H}$ each isomer), 5.82 (br s, $1 \mathrm{H}$ each isomer), 6.92-7.07 (m), 7.12 (t, $J=7.5 \mathrm{~Hz}, 1 \mathrm{H}$ each isomer), 7.21 (t, $J=7.5 \mathrm{~Hz}$, $1 \mathrm{H}$ each isomer), 7.35 (d, $J=7.5 \mathrm{~Hz}, 1 \mathrm{H}$ each isomer), $7.77(\mathrm{~d}, J=7.5 \mathrm{~Hz}, 1 \mathrm{H}), 7.80(\mathrm{~d}, J=$ $7.5 \mathrm{~Hz}, 1 \mathrm{H}$ ), 8.20 (br s, $1 \mathrm{H}$ each isomer); ${ }^{13} \mathrm{C} \mathrm{NMR}\left(\mathrm{CDCl}_{3}, 75 \mathrm{MHz}\right.$, major epimer) $\delta 21.8$ $\left(\mathrm{CH}_{3}\right), 30.6\left(\mathrm{CH}_{2}\right), 32.8\left(\mathrm{CH}_{2}\right), 50.4\left(\mathrm{CH}_{2}\right), 53.4\left(\mathrm{CH}_{2}\right), 55.3(\mathrm{CH}), 70.4(\mathrm{CH}), 111.2(\mathrm{CH}), 116.6$ (C), $119.5(\mathrm{CH}), 1119.8(\mathrm{CH}), 120.2(\mathrm{CH}), 122.1(\mathrm{CH}), 122.3(\mathrm{CH}), 125.2(\mathrm{CH}), 126.8(\mathrm{C})$, $128.0(2 \mathrm{CH}), 128.6(2 \mathrm{CH}), 136.2(\mathrm{C}), 139.4(\mathrm{C}), 139.5(\mathrm{C}) ;{ }^{13} \mathrm{C} \mathrm{NMR}\left(\mathrm{CDCl}_{3}, 75 \mathrm{MHz}\right.$, minor epimer) $\delta 21.6\left(\mathrm{CH}_{3}\right), 30.8\left(\mathrm{CH}_{2}\right), 32.6\left(\mathrm{CH}_{2}\right), 50.3\left(\mathrm{CH}_{2}\right), 53.2\left(\mathrm{CH}_{2}\right), 54.8(\mathrm{CH}), 70.0(\mathrm{CH})$, $111.2(\mathrm{CH}), 116.3(\mathrm{C}), 119.5(\mathrm{CH}), 119.8(\mathrm{CH}), 120.2(\mathrm{CH}), 122.1(\mathrm{CH}), 122.2(\mathrm{CH}), 125.3$ $(\mathrm{CH}), 126.9(\mathrm{C}), 128.2(2 \mathrm{CH}), 128.6(2 \mathrm{CH}), 136.1(\mathrm{C}), 139.4(\mathrm{C}), 139.5(\mathrm{C})$. Exact mass Calcd for $\mathrm{C}_{23} \mathrm{H}_{26} \mathrm{~N}_{2} \mathrm{OS}: 378.1765$. Found: 378.1754 .

Methyl 5(Z)-ethylidene-2-(3-indolyl)-1-[2-(phenylsulfanyl)ethyl]-4-piperidineacetate (mixture of isomers) (10). Operating as described for 6, starting from tetrahydropyridine 9 (370 $\mathrm{mg}$, $0.98 \mathrm{mmol}$, mixture of epimers), 4-piperidineacetate 10 (292 $\mathrm{mg}, 69 \%)$ was obtained after chromatography ( $7: 3$ hexane-AcOEt) as a mixture of cis and trans isomers, which could not be separated. IR (film) 3300, 1600, 1450, $668 \mathrm{~cm}^{-1} ;{ }^{1} \mathrm{H} \mathrm{NMR}\left(\mathrm{CDCl}_{3}, 300 \mathrm{MHz}\right.$, most significant signals from a mixture of isomers) $\delta 1.66(\mathrm{~d}, J=6.5 \mathrm{~Hz}, 3 \mathrm{H}), 1.73(\mathrm{~d}, J=6.5 \mathrm{~Hz}, 3 \mathrm{H}), 1.92(\mathrm{~d}$, $J=12.5 \mathrm{~Hz}, 1 \mathrm{H}), 2.56$ (dd, $J=14.4,7.5 \mathrm{~Hz}, 1 \mathrm{H}), 3.65(\mathrm{~s}, 3 \mathrm{H}), 3.67$ (s, $3 \mathrm{H}), 3.77$ (d, $J=13.2$ $\mathrm{Hz}, 1 \mathrm{H}), 3.78$ (dd, $J=11.5,2.8 \mathrm{~Hz}, 1 \mathrm{H}), 3.83$ (dd, $J=11.5,2.8 \mathrm{~Hz}, 1 \mathrm{H}), 4.03$ (d, $J=12.0 \mathrm{~Hz}$, $1 \mathrm{H}$ each isomer), $5.20(\mathrm{q}, J=6.8 \mathrm{~Hz}, 1 \mathrm{H}), 5.43(\mathrm{q}, J=6.8 \mathrm{~Hz}, 1 \mathrm{H}), 6.90-7.00(\mathrm{~m}), 7.10(\mathrm{t}, J=$ $7.5 \mathrm{~Hz}, 1 \mathrm{H}$ each isomer), 7.20 (t, $J=7.5 \mathrm{~Hz}, 1 \mathrm{H}$ each isomer), 7.35 (d, $J=7.5 \mathrm{~Hz}, 1 \mathrm{H}$ each 
isomer), 7.85 (dm, $J=7.5 \mathrm{~Hz}, 1 \mathrm{H}$ each isomer), 8.15 (br s, $1 \mathrm{H}$ each isomer); ${ }^{13} \mathrm{C} \mathrm{NMR}\left(\mathrm{CDCl}_{3}\right.$, $75 \mathrm{MHz}$, signals of cis isomer from an enriched fraction) $\delta 13.1\left(\mathrm{CH}_{3}\right), 30.5\left(\mathrm{CH}_{2}\right), 36.9\left(\mathrm{CH}_{2}\right)$, $38.9(\mathrm{CH}), 41.4\left(\mathrm{CH}_{2}\right), 51.6\left(\mathrm{CH}_{3}\right), 53.0\left(\mathrm{CH}_{2}\right), 53.2\left(\mathrm{CH}_{2}\right), 60.4(\mathrm{CH}), 111.1(\mathrm{CH}), 115.0(\mathrm{CH})$, $118.0(\mathrm{C}), 119.4(\mathrm{CH}), 120.2(\mathrm{CH}), 122.1(\mathrm{CH}), 122.2(\mathrm{CH}), 125.0(\mathrm{CH}), 126.4(\mathrm{C}), 127.7$ (2

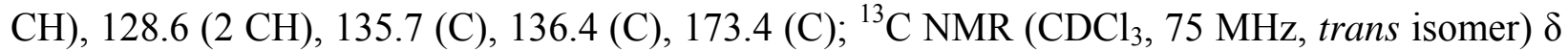
$13.1\left(\mathrm{CH}_{3}\right), 30.6\left(\mathrm{CH}_{2}\right), 37.9\left(\mathrm{CH}_{2}\right), 38.1\left(\mathrm{CH}_{2}\right), 39.3(\mathrm{CH}), 48.6\left(\mathrm{CH}_{2}\right), 51.6\left(\mathrm{CH}_{3}\right), 53.1\left(\mathrm{CH}_{2}\right)$, $55.1(\mathrm{CH})$. Anal. Calcd for $\mathrm{C}_{26} \mathrm{H}_{30} \mathrm{~N}_{2} \mathrm{O}_{2} \mathrm{~S} \cdot 1 / 2 \mathrm{H}_{2} \mathrm{O}$ : C, 67.65; H, 7.21; N, 6.11. Found: C, 67.99; $\mathrm{H}, 6.92 ; \mathrm{N}, 6.06$.

Methyl 5(Z)-ethylidene-2-[1-(methoxycarbonyl)-3-indolyl]-1-[2-(phenylsulfanyl)ethyl]-4-piperidine acetate (mixture of epimers) (11). Operating as described for 7, starting from 10 (225 mg, $0.52 \mathrm{mmol}$, mixture of isomers), compound 11 (190 mg, 74\%) was obtained as a mixture of cis and trans isomers after column chromatography (7:3 hexane-AcOEt). IR (film) 3950, 1737, $1455,1258,731 \mathrm{~cm}^{-1}$; ${ }^{1} \mathrm{H} \mathrm{NMR}\left(\mathrm{CDCl}_{3}, 300 \mathrm{MHz}\right.$, most significant signals from a mixture of isomers) $\delta 1.65(\mathrm{~d}, J=6.6 \mathrm{~Hz}, 3 \mathrm{H}), 1.72(\mathrm{~d}, J=6.6 \mathrm{~Hz}, 3 \mathrm{H}), 1.87(\mathrm{dm}, J=12.6 \mathrm{~Hz}, 1 \mathrm{H}), 2.23$ $(\mathrm{dd}, J=14.1,7.8 \mathrm{~Hz}, 1 \mathrm{H}), 3.63(\mathrm{~s}, 3 \mathrm{H}), 3.64$ (s, $3 \mathrm{H}), 3.76(\mathrm{~d}, J=12.3 \mathrm{~Hz}, 1 \mathrm{H}), 3.77$ (dd, $J=$ 11.5, $2.8 \mathrm{~Hz}, 1 \mathrm{H}), 3.80(\mathrm{dd}, J=11.5,2.8 \mathrm{~Hz}, 1 \mathrm{H}), 3.90(\mathrm{~d}, J=12.3 \mathrm{~Hz}, 1 \mathrm{H}), 4.00$ (s, $3 \mathrm{H}), 4.01$ $(\mathrm{s}, 3 \mathrm{H}), 5,20(\mathrm{q}, J=6.6 \mathrm{~Hz}, 1 \mathrm{H}), 5.43(\mathrm{q}, J=6.6 \mathrm{~Hz}, 1 \mathrm{H}), 6.80-7.00(\mathrm{~m}), 7,24(\mathrm{t}, J=7.5 \mathrm{~Hz}, 1$ $\mathrm{H}$ each isomer), $7.36(\mathrm{t}, J=7.5 \mathrm{~Hz}, 1 \mathrm{H}$ each isomer), $7.44(\mathrm{~d}, J=7.5 \mathrm{~Hz}, 1 \mathrm{H}$ each isomer), 7.86 $(\mathrm{d}, J=7.5 \mathrm{~Hz}, 1 \mathrm{H}), 7.88(\mathrm{~d}, J=7.5 \mathrm{~Hz}, 1 \mathrm{H}) 8.20\left(\mathrm{~d}, J=7.5 \mathrm{~Hz}, 1 \mathrm{H}\right.$ each isomer); ${ }^{13} \mathrm{C}$ NMR $\left(\mathrm{CDCl}_{3}, 75 \mathrm{MHz}\right.$, signals of $c$ is isomer from an enriched fraction) $\delta 12.9\left(\mathrm{CH}_{3}\right), 30.5\left(\mathrm{CH}_{2}\right), 36.6$ $\left(\mathrm{CH}_{2}\right), 38.5(\mathrm{CH}), 40.1\left(\mathrm{CH}_{2}\right), 51.5\left(\mathrm{CH}_{3}\right), 52.7\left(\mathrm{CH}_{2}\right), 52.9\left(\mathrm{CH}_{2}\right), 53.5\left(\mathrm{CH}_{3}\right), 60.1(\mathrm{CH}), 114.9$ $(\mathrm{CH}), 120.0(\mathrm{CH}), 122.6(\mathrm{CH}), 122.8(\mathrm{CH}), 123.0(\mathrm{C}), 124.6(\mathrm{CH}), 125.2(\mathrm{CH}), 127.8(\mathrm{CH})$, $128.0(2 \mathrm{CH}), 128.3(2 \mathrm{CH}), 128.9(\mathrm{C}), 135.0(\mathrm{C}), 135.8(\mathrm{C}), 136.5(\mathrm{C}), 151.0(\mathrm{C}), 173.0(\mathrm{C}) ;{ }^{13} \mathrm{C}$ NMR $\left(\mathrm{CDCl}_{3}, 75 \mathrm{MHz}\right.$, trans isomer) $\delta 12.8\left(\mathrm{CH}_{3}\right), 30.7\left(\mathrm{CH}_{2}\right), 36.9\left(\mathrm{CH}_{2}\right), 37.7\left(\mathrm{CH}_{2}\right), 38.9$ $(\mathrm{CH}), 48.2\left(\mathrm{CH}_{2}\right), 51.4\left(\mathrm{CH}_{3}\right), 52.8\left(\mathrm{CH}_{2}\right), 53.6\left(\mathrm{CH}_{3}\right), 55.0(\mathrm{CH})$. Anal. Calcd for $\mathrm{C}_{28} \mathrm{H}_{32} \mathrm{~N}_{2} \mathrm{O}_{4} \mathrm{~S} \cdot 2 / 3 \mathrm{H}_{2} \mathrm{O}: \mathrm{C}, 66.64 ; \mathrm{H}, 6.66 ; \mathrm{N}, 5.55$. Found: C, 66.44; H, 6.34; N, 5.77.

\section{Methyl 5(Z)-ethylidene-2-[1-(methoxycarbonyl)-3-indolyl]-1-[2-(phenylsulfinyl)ethyl]-4-piperidine} acetate (mixture of isomers) (12). TFA (38 $\mu \mathrm{L}, 0.49 \mathrm{mmol})$ was added to a solution of sulfide 11 (190 mg, $0.38 \mathrm{mmol})$ in anhydrous $\mathrm{CH}_{2} \mathrm{Cl}_{2}(15 \mathrm{~mL})$ at $0{ }^{\circ} \mathrm{C}$, and the mixture was stirred for $30 \mathrm{~min}$. The temperature was lowered to $-60^{\circ} \mathrm{C}$, a solution of $\mathrm{m}$-CPBA ( $\left.79 \mathrm{mg}, 0.46 \mathrm{mmol}\right)$ in anhydrous $\mathrm{CH}_{2} \mathrm{Cl}_{2}(3 \mathrm{~mL})$ was slowly added, and the stirring was continued for $2 \mathrm{~h}$. Then, solid $\mathrm{K}_{2} \mathrm{CO}_{3}(0.5 \mathrm{~g})$ was added, and the mixture was stirred for $2 \mathrm{~h}$ at room temperature, filtered, washed with brine, and concentrated. Column chromatography $\left(\mathrm{Et}_{2} \mathrm{O}\right)$ of the residue afforded sulfoxide 12 (142 mg, 72\%) as a mixture of $\mathrm{S}=\mathrm{O}$ and 2,4-cis/trans isomers. IR (film) 3400, 1596, 1578, 1459, $668 \mathrm{~cm}^{-1} ;{ }^{1} \mathrm{H}$ NMR $\left(\mathrm{CDCl}_{3}, 300 \mathrm{MHz}\right.$, most significant signals); $\delta 1.63$ (d, J=6.6 $\mathrm{Hz}, 3 \mathrm{H}), 1.67(\mathrm{~d}, J=6.6 \mathrm{~Hz}, 3 \mathrm{H}), 1.70(\mathrm{~d}, J=6.6 \mathrm{~Hz}, 3 \mathrm{H}), 1.73(\mathrm{~d}, J=6.6 \mathrm{~Hz}, 3 \mathrm{H}), 3.63(\mathrm{~s}, 3$ $\mathrm{H}), 3.64(\mathrm{~s}, 3 \mathrm{H}), 3.65(\mathrm{~s}, 3 \mathrm{H}), 3.66(\mathrm{~s}, 3 \mathrm{H}), 4.03\left(\mathrm{~s}\right.$, corresponding to $\left.4 \mathrm{OCH}_{3}\right), 5.23(\mathrm{q}, J=6.6$ $\mathrm{Hz}$, corresponding to $2=\mathrm{CH}$ ), 5.43 (c, $J=6.6 \mathrm{~Hz}$, corresponding to $2=\mathrm{CH}$ ). Anal. Calcd for $\mathrm{C}_{28} \mathrm{H}_{32} \mathrm{~N}_{2} \mathrm{O}_{5} \mathrm{~S} \cdot 1 / 2 \mathrm{H}_{2} \mathrm{O}: \mathrm{C}, 64.97 ; \mathrm{H}, 6.43 ; \mathrm{N}, 5.41$. Found: C, 64.95; H, 6.32; N, 5.41. 
Methyl 3(Z)-ethylidene-8-(methoxycarbonyl)-7-(phenylsulfanyl)indolo[3,2-a]quinolizidine2-acetate (13). Diisopropylethylamine (DIPEA) $(130 \mu \mathrm{L}, 0.76 \mathrm{mmol})$ and trimethylsilyl triflate $(140 \mu \mathrm{L}, 0.76 \mathrm{mmol})$ were added to a $0{ }^{\circ} \mathrm{C}$ cooled solution of sulfoxide 12 (100 $\left.\mathrm{mg}, 0.19 \mathrm{mmol}\right)$ in anhydrous $\mathrm{CH}_{2} \mathrm{Cl}_{2}(8 \mathrm{~mL})$, and the mixture was stirred at room temperature for $1 \mathrm{~h} 30 \mathrm{~min}$. The resulting reaction mixture was diluted with $\mathrm{CH}_{2} \mathrm{Cl}_{2}(10 \mathrm{~mL})$, washed with saturated aqueous $\mathrm{NaHCO}_{3}$, dried, filtered, and concentrated. The residue, after purification by chromatography (9:1 $\mathrm{CH}_{2} \mathrm{Cl}_{2}$-AcOEt), afforded quinolizidine $13(40 \mathrm{mg}, 42 \%$ ) as a mixture of isomers 13a and 13b, which could not be separated. MS m/e (relative intensity): $490\left(\mathrm{M}^{+}, 8\right), 381\left(\mathrm{M}^{+}-\mathrm{C}_{6} \mathrm{H}_{5} \mathrm{~S}\right.$, 100), 307 (21), 227 (27), 168 (21), 110 (21); cis isomer: ${ }^{1} \mathrm{H} \mathrm{NMR} \mathrm{(} \mathrm{CDCl}_{3}, 500 \mathrm{MHz}$, assigned by HSQC and COSY experiments): $\delta 1.41(\mathrm{q}, J=12.5 \mathrm{~Hz}, 1 \mathrm{H}, \mathrm{H}-1), 1.54(\mathrm{~d}, J=6.8 \mathrm{~Hz}, 3 \mathrm{H}$, $\left.\mathrm{CH}_{3}\right), 2.14(\mathrm{dm}, J=12.5 \mathrm{~Hz}, 1 \mathrm{H}, \mathrm{H}-1), 2.64\left(\mathrm{dd}, J=14.5,8.0 \mathrm{~Hz}, 1 \mathrm{H}, \mathrm{CH}_{2} \mathrm{CO}\right), 2.74(\mathrm{dd}, J=$ 14.5, 8.2 Hz, $\left.1 \mathrm{H}, \mathrm{CH}_{2} \mathrm{CO}\right), 2.76$ (dd, $\left.J=12.3,3.3 \mathrm{~Hz}, 1 \mathrm{H}, \mathrm{H}-6\right), 2.85$ (m, $\left.1 \mathrm{H}, \mathrm{H}-2\right), 3.40$ (d, $J$ $=11.5 \mathrm{~Hz}, \mathrm{H}-4), 3.42(\mathrm{dd}, J=12.3,3.6 \mathrm{~Hz}, 1 \mathrm{H}, \mathrm{H}-6), 3.62\left(\mathrm{~s}, 3 \mathrm{H}, \mathrm{OCH}_{3}\right), 3.78\left(\mathrm{~s}, 3 \mathrm{H}, \mathrm{OCH}_{3}\right)$, 3.81 (d, $J=11.5 \mathrm{~Hz}, 1 \mathrm{H}, \mathrm{H}-4), 4.25$ (dd, $J=12.5,3.5 \mathrm{~Hz}, 1 \mathrm{H}, \mathrm{H}-12 \mathrm{c}$ ), 4.99 (br s, $1 \mathrm{H}, \mathrm{H}-7$ ), $5.46(\mathrm{q}, J=6.8 \mathrm{~Hz}, 1 \mathrm{H},=\mathrm{CH}), 7.21-7.46(\mathrm{~m}, 8 \mathrm{H}, \mathrm{Ar}), 8.11(\mathrm{~d}, J=7.5 \mathrm{~Hz}, \mathrm{H}-9) .{ }^{13} \mathrm{C}$ NMR $\left(\mathrm{CDCl}_{3}, 75 \mathrm{MHz}\right.$, assigned by HSQC experiments): $\delta 12.7\left(\mathrm{CH}_{3}\right), 36.2(\mathrm{C}-1), 37.8\left(\mathrm{CH}_{2} \mathrm{CO}\right)$, 38.5 (C-2), $45.0(\mathrm{C}-7), 51.5\left(\mathrm{OCH}_{3}\right), 52.0(\mathrm{C}-6), 53.5\left(\mathrm{OCH}_{3}\right), 54.6(\mathrm{C}-4), 54.9(\mathrm{C}-12 \mathrm{c}), 115.6$ (C-12b), 115.7 (C-9), 116.0 (=CH), 118.6 (C-12), 123.0 (C-11), 124.8 (C-10), 127.0 (C-12a), $127.1(\mathrm{C}-p), 128.9(\mathrm{C}-m), 131.7(\mathrm{C}-o), 136.0(\mathrm{C}-3), 151.7(\mathrm{C}=\mathrm{O}), 172.7(\mathrm{C}=\mathrm{O})$. trans isomer: ${ }^{1} \mathrm{H}$ NMR ( $\mathrm{CDCl}_{3}, 500 \mathrm{MHz}$, assigned by HSQC and COSY experiments): $\delta 1.60$ (d, J=6.8 Hz, 3 $\mathrm{H}, \mathrm{CH}_{3}$ ), 1.94 (m, $2 \mathrm{H}, \mathrm{H}-1$ ), 2.22 (dd, $J=15.5,7.0 \mathrm{~Hz}, 1 \mathrm{H}, \mathrm{CH}_{2} \mathrm{CO}$ ), 2.61 (dd, J=15.5, 6.5 Hz, $1 \mathrm{H}, \mathrm{CH}_{2} \mathrm{CO}$ ), 2.73 (dd, $\left.J=12.0,5.0 \mathrm{~Hz}, 1 \mathrm{H}, \mathrm{H}-6\right), 2.85$ (m, $\left.1 \mathrm{H}, \mathrm{H}-2\right), 3.50$ (dd, $J=12.0,3.6$ $\mathrm{Hz}, 1 \mathrm{H}, \mathrm{H}-6), 3.56$ (m, $2 \mathrm{H}, \mathrm{H}-4), 3.65$ (s, $\left.3 \mathrm{H}, \mathrm{OCH}_{3}\right), 3.78$ (s, $\left.3 \mathrm{H}, \mathrm{OCH}_{3}\right), 4.20$ (dd, J = 12.0, $3.0 \mathrm{~Hz}, 1 \mathrm{H}, \mathrm{H}-12 \mathrm{c}$ ), 4.99 (br s, $1 \mathrm{H}, \mathrm{H}-7), 5.25$ (q, $J=6.8 \mathrm{~Hz}, 1 \mathrm{H},=\mathrm{CH}), 7.21-7.46$ (m, $8 \mathrm{H}$, $\mathrm{Ar}), 8.11(\mathrm{~d}, J=7.5 \mathrm{~Hz}, \mathrm{H}-9) .{ }^{13} \mathrm{C} \mathrm{NMR}\left(\mathrm{CDCl}_{3}, 75 \mathrm{MHz}\right.$, most significant signals assigned by HSQC experiments): $\delta 13.0\left(\mathrm{CH}_{3}\right), 32.9(\mathrm{C}-1), 36.9\left(\mathrm{CH}_{2} \mathrm{CO}\right), 40.1(\mathrm{C}-2), 45.2(\mathrm{C}-7), 49.8(\mathrm{C}-4)$, $50.3(\mathrm{C}-12 \mathrm{c}), 51.5\left(\mathrm{OCH}_{3}\right), 52.4(\mathrm{C}-6), 53.5\left(\mathrm{OCH}_{3}\right), 121.5(=\mathrm{CH}), 135.5(\mathrm{C}-3), 151.7(\mathrm{C}=\mathrm{O})$, $173.0(\mathrm{C}=\mathrm{O})$.

\section{Acknowledgments}

This work was supported by the DGICYT, Spain (BQU2000-0651). Thanks are also due to the DURSI, Generalitat de Catalunya, for Grant 2001SGR-0084 and a fellowship to M.-D. C., and to the SCT of the University of Barcelona for recording the NMR and mass spectra. 


\section{References and Notes}

1. (a) Amat, M.; Llor, N.; Pshenichnyi, G.; Bosch, J. ARKIVOC 2002, (iii), 73. (b) Amat, M.; Seffar, F.; Llor, N.; Bosch, J. Synthesis 2001, 267. (c) Amat, M.; Hadida, S.; Pshenichnyi, G.; Bosch, J. J. Org. Chem. 1997, 62, 3158, and references cited therein.

2. (a) Bosch, J.; Bonjoch, J.; Amat, M. In The Alkaloids; Cordell, G. A., Ed.; Academic Press: San Diego, 1996; Vol. 48, Chapter 2. (b) Sapi, J.; Massiot, G. In Monoterpenoid Indole Alkaloids; Saxton, J. E., Ed. In The Chemistry of Heterocyclic Compounds; Taylor, E. C., Ed.; Wiley: Chichester, 1994; Supplement to Vol. 25, Part 4, Chapter 7. (c) Husson, H.-P. In The Monoterpenoid Indole Alkaloids; Saxton, J. E., Ed. In The Chemistry of Heterocyclic Compounds; Weissberger, A.; Taylor, E. C., Eds.; Wiley: Chichester, 1983; Vol. 25, Part 4, Chapter VII.

3. Amat, M.; Bosch, J. J. Org. Chem. 1992, 57, 5792.

4. For the use of TMSOTf as initiator of Pummerer reactions, see: (a) García-Ruano, J. L.; García-Paredes, C. Tetrahedron Lett. 2000, 41, 261. (b) Padwa, A.; Waterson, A. G. Tetrahedron 2000, 56, 10159. (c) Craig, D.; Daniels, K.; MacKenzie, A. R. Tetrahedron 1992, 48, 7803, and references cited therein.

5. For a review on the use of the Pummerer reaction in the synthesis of heterocycles, see: (a) Padwa, A.; Gunn, D. E., Jr.; Osterhout, M. H. Synthesis 1997, 1353. For other reviews, see: (b) DeLucchi, O.; Miotti, U.; Modena, G. Organic Reactions 1991, 40, 157. (c) Grierson, D. S.; Husson, H.-P. In Comprehensive Organic Synthesis; Trost, B. M., Fleming, I., Eds.; Pergamon Press: Oxford, 1991; Vol. 6, pp 907-947. (d) Kennedy, M.; McKervey, M. A. In Comprehensive Organic Synthesis; Trost, B. M., Fleming, I., Eds.; Pergamon Press: Oxford, 1991; Vol. 7, pp 193-216.

6. (a) Emde, H.; Simchen, G. Liebigs Ann. Chem. 1983, 816. See also: (b) Brownbridge, P. Synthesis 1983, 1. (c) Brownbridge, P. Synthesis 1983, 85.

7. (a) Remers, W. A. In Indoles; Houlihan, W. J., Ed. In The Chemistry of Heterocyclic Compounds; Weissberger, A.; Taylor, E. C., Eds.; Wiley: New York, 1972; Part 1, Chapter I; (b) Sundberg, R. J. The Chemistry of Indoles; Blomquist, A. T., Ed.; Academic Press: New York, 1970; Chapter I.

8. (a) Biswas, K. M.; Jackson, A. H. Tetrahedron 1969, 25, 227. (b) Jackson, A. H.; Naidoo, B.; Smith, P. Tetrahedron 1968, 24, 6119. (c) Jackson, A. H.; Smith, P. Tetrahedron 1968, 24, 2227.

9. (a) Cox, E. D.; Cook, J. M. Chem. Rev. 1995, 95, 1797. (b) Kowalski, P.; Bojarski, A. J.; Mokrosz, J. L. Tetrahedron 1995, 51, 2737, and references cited therein.

10. (a) Frost, J. R.; Gaudillière, B. R. P.; Kauffmann, E.; Loyaux, D.; Normand, N.; Petry, G.; Poirier, P.; Wenkert, E.; Wick, A. E. Heterocycles 1989, 28, 175. (b) Biswas, K. M.; Dhara, R. N.; Halder, S.; Mallik, H.; Sinha-Chaudhuri, A.; De, P.; Brahmachari, A. S. Synth. Commun. 1993, 23, 379. 
11. For a review: (a) Ungemach, F.; Cook, J. M. Heterocycles 1978, 9, 1089. See also: (b) Ganesan, A.; Heathcok, C. H. Tetrahedron Lett. 1993, 34, 439. (c) Curness, K.; Dyke, S. Heterocycles 1979, 12, 1133.

12. For mechanistic studies in the context of the synthesis of eudistomins, see: (a) Liu, J.-J.; Hino, T.; Tsuruoka, A.; Harada, N.; Nakagawa, M. J. Chem. Soc., Perkin Trans. 1 2000, 3487. (b) van Maarseveen, J. H.; Scheeren, W.; Kruse, C. G. Tetrahedron 1993, 49, 2325. (c) Kawate, T.; Nakagawa, M.; Ogata, K.; Hino, T. Heterocycles 1992, 33, 801. (d) Liu, J.; Nakagawa, M.; Ogata, K.; Hino, T. Chem. Pharm. Bull. 1991, 39, 1672. (e) Liu, J.; Nakagawa, M.; Hino, T. Tetrahedron 1989, 45, 7729. (f) Nakagawa, M.; Liu, J.; Ogata, K.; Hino, T. J. Chem. Soc., Chem. Commun. 1988, 463. For the intramolecular trapping of the indolenine intermediate by enol or enolates, see: (g) Turet, L.; Markó, I. E.; Tinant, B.; Declercq, J.-P.; Touillaux, R. Tetrahedron Lett. 2002, 43, 6591 (h) England, D. B.; Kuss, T. D. O.; Keddy, R. G.; Kerr, M. J. Org. Chem. 2001, 66, 4704. (i) Bobowski, G.; Morrison, G. C. J. Org. Chem. 1981, 46, 4927. (j) Garnick, R. L.; Levery, S. B.; Le Quesne, P. W. J. Org. Chem. 1978, 43, 1226. (k) Büchi, G.; Matsumoto, K. E.; Nishimura, H. J. Am. Chem. Soc. 1971, 93, 3299. For the intramolecular trapping of the indolenine intermediate by an allylsilane, see: (1) He, F.; Bo, Y.; Altom, J. D.; Corey, E. J. J. Am. Chem. Soc. 1999, 121, 6771.

13. For the intramolecular trapping of the spiroindoleninium salt generated in a Pummerer reaction from a 3-substituted indole, see: Oikawa, Y.; Yonemitsu, O. J. Org. Chem. 1976, 41, 1118.

14. Diez, A.; Vila, C.; Sinibaldi, M.-E.; Troin, Y.; Rubiralta, M. Tetrahedron Lett. 1993, 34, 733.

15. For reviews, see: (a) Rhoads, S. J.; Raulins, N. R. Org. React. 1975, 22, 1. (b) Wipf, P. In Comprehensive Organic Synthesis; Trost, B. M., Ed.; Pergamon Press: Oxford, 1991. Vol. 5, Chapter 7.

16. This stereochemical issue did not concern us unduly because the reversibility of spiroindolenine intermediates related to $\mathbf{B}$ (Figure 1) through the corresponding iminium salts has been postulated: Melnyk, P.; Ducrot, P.; Thal, C. Tetrahedron 1993, 49, 8589, and references cited therein.

17. Casnati, G.; Dossena, A.; Pochini, A. Tetrahedron Lett. 1972, 5277.

18. (a) Wenkert, E.; Shi, Y.-J. Synth. Commun. 1989, 19, 1071. (b) Finch, N.; Gemenden, C. W.; Hsu, I. H.-C.; Kerr, A.; Sim, G. A.; Taylor, W. I. J. Am. Chem. Soc. 1965, 87, 2229. 